Revue

de |'histoire des religions

\section{Revue de l'histoire des religions}

$2 \mid 2015$

Les images miraculeuses de la Vierge au premier âge moderne entre dévotion locale et culte universel

\title{
Vivantes ou comme vivantes : l'animation miraculeuse d'images de la Vierge entre Moyen Âge et époque moderne
}

Living or as if living: the miraculous animation of images of the Virgin between

the Middle Ages and Modern Times

Jean-Marie Sansterre

\section{OpenEdition}

Journals

Édition électronique

URL : http://journals.openedition.org/rhr/8393

DOI : 10.4000/rhr.8393

ISSN : 2105-2573

Éditeur

Armand Colin

Édition imprimée

Date de publication : 1 juin 2015

Pagination : 155-182

ISBN : 978-2-200-92965-7

ISSN : 0035-1423

Référence électronique

Jean-Marie Sansterre, «Vivantes ou comme vivantes : I'animation miraculeuse d'images de la Vierge entre Moyen Âge et époque moderne », Revue de l'histoire des religions [En ligne], 2 | 2015, mis en ligne le 01 juin 2018, consulté le 22 avril 2019. URL : http://journals.openedition.org/rhr/8393 ; DOI

$10.4000 /$ rhr.8393 


\section{Vivantes ou comme vivantes : l'animation miraculeuse d'images de la Vierge entre Moyen Âge et époque moderne}

L'histoire de l'animation miraculeuse des images s'inscrit dans la longue durée. Centré sur les effigies de la Vierge, l'article évoque d'abord deux cas de la fin du XVIII siècle; puis il remonte aux siècles précédents pour un bref parcours entre Moyen Âge et époque moderne. Si les récits miraculaires sont souvent peu contextualisés avant le milieu du XIII siècle, il y a de notables exceptions qui deviennent ensuite la règle. Les miracles d'images «vivantes» se multiplient alors, tant dans les expériences mystiques que dans leurs formes publiques et collectives, qui retiennent ici particulièrement l'attention. L'article met ainsi en évidence un ensemble d'attitudes et de croyances qui intéressent in fine la question fondamentale des rapports entre le personnage céleste et sa représentation.

\section{Living or as if living: the miraculous animation of images of the Virgin between the Middle Ages and Modern Times}

The history of the miraculous animation of images was long lasting. This article, which focuses on effigies of the Virgin, first evokes two cases from the late $18^{\text {th }}$ century; it then goes back to the preceding centuries, offering a brief journey from the Middle Ages up to Modern Times. While accounts of these miracles are rarely contextualised before the mid$13^{\text {th }}$ century, there are notable exceptions that later become the rule. The miracles produced by "living" images then proliferated both as mystical experiences as well as in their public and collective forms, which are given particular attention here. The article thus highlights a set of attitudes and beliefs that are ultimately related to the fundamental issue of the relationship between the celestial figure and her representation. 
Bien que leurs mentions soient particulièrement nombreuses du $\mathrm{XIV}^{\mathrm{e}}$ au $\mathrm{XVII}^{\mathrm{e}}$ siècle, l'histoire des images «vivantes» du christianisme occidental s'inscrit dans la longue durée. Comme je l'ai montré tout récemment, divers textes des $\mathrm{X}^{\mathrm{e}}-\mathrm{XIII}^{\mathrm{e}}$ siècles infirment la chronologie basse proposée à ce propos par Caroline Walker Bynum ${ }^{1}$. J'aimerais revenir sur le sujet en privilégiant ici les images de la Vierge et en menant l'exposé jusqu'à la fin du XVIII ${ }^{\mathrm{e}}$ siècle. Si les miracles d'animation d'images - ou du moins leur reconnaissance - semblent s'être raréfiés durant ce siècle, ils connurent tout à la fin un extraordinaire renouveau dans l'État de l'Église. On commencera par lui parce qu'il a fait l'objet d'une approche historienne exemplaire et parce que les textes permettent quelques observations sur les rapports, lors du miracle, entre l'image et son prototype céleste, un aspect important pour notre propos que l'approche en question n'envisage pas. On s'arrêtera ensuite à une mention peu connue concernant un épisode de la Révolution brabançonne à la même époque. Puis, dans une brève esquisse, on ira en amont, du Moyen Âge à l'époque moderne².

\section{À LA FIN DU XVIII ${ }^{\mathrm{E}}$ SIÈCLE, DeS MIRACLES D'IMAGES DANS L'ÉTAT PONTIFICAL MENACÉ ET DANS LE BRABANT EN RÉVOLTE}

Nous sommes en 1796 et 1797 dans l'État pontifical. En juin 1796, l'armée française s'est emparée de Ferrare et de Bologne, une situation reconnue par l'armistice conclu quelques jours plus tard, le 23 juin. Dans le reste de l'État de l'Église, la peur à l'égard des troupes de la République se propagea avec d'autant plus de force qu'une active

1. Jean-Marie Sansterre, «La imagen activada por su prototipo celestial: milagros occidentales anteriores a mediados del siglo XIII », Codex Aquilarensis, 29, 2013, p. 77-98. Contra: Caroline Walker Bynum, Christian Materiality. An Essay on Religion in Late Medieval Europe, New York, Zone Books, 2011, p. 107-112, 323-327. Sur cet ouvrage important, cf. infra n. 46.

2. Version remaniée du texte lu lors de la journée d'étude du Gemca (Centre d'analyse culturelle de la première modernité, Université catholique de Louvain) le 6 mars 2012, le présent article garde son caractère de simple esquisse. En outre, le nombre de pages imparti ne permet pas de reprendre les textes originaux en plus des traductions. 
propagande avait donné une image terrifiante des révolutionnaires français. Le 25 juin, devant de nombreux fidèles, une Vierge peinte vénérée dans la cathédrale d'Ancône ouvrit grand les yeux qu'elle avait entrouverts et, de la bouche, elle esquissa un sourire. Ce prodige fut le point de départ d'une extraordinaire vague de miracles du même genre qui se répandit dans l'État pontifical jusque vers février 1797, le phénomène faisant l'objet à l'époque d'une lecture souvent optimiste, un signe de réconfort et non pas l'annonce de malheurs. Au début, la vague toucha aussi des images de sainte Anne, de saints locaux et du Christ et se manifesta sous des formes variées - mouvements des yeux, mouvements des bras, larmes, sueurs, «convulsions», changements de couleur -, puis la vague de miracles se centra presque exclusivement sur des images de la Vierge selon une typologie désormais presque uniformisée: elle ne concerna généralement plus que les yeux, soit ouverture et fermeture des paupières, soit mouvement vertical ou horizontal des pupilles. C'est ce qui arriva à Rome à quantité de madones, peintures sur toile, fresques et parfois une statue ou statuette, images plus ou moins vénérées placées en de multiples endroits, églises, chapelles, édicules dans les rues et maisons privées. Les prodiges observés sur 24 d'entre elles ainsi que sur deux figures du Christ en croix furent officiellement déclarés authentiques le 28 février 1797, après des procès canoniques limités, pour ne pas prendre trop de temps, à une partie des images miraculeuses.

Un passionnant ouvrage de Massimo Cattaneo publié en 1995 a mis en œuvre les pièces de ces procès canoniques et de ceux menés dans d'autres évêchés ainsi que de nombreuses autres sources ${ }^{3}$. Le livre, qui bien sûr examine aussi les témoignages plus rares de scepticisme ou d'hostilité, offre un exemple remarquable d'une approche du miracle dans «sa dimension d'événement historique», une approche dont Sofia Boesh a maintes fois montré l'intérêt et la nécessitét. On ne peut que souscrire à une réflexion comme celle-ci :

De façon générale, je crois que l'on doit parler pour la «vague miraculeuse» de 1796-97 de «bonne foi», sûrement de la part du

3. Massimo Cattaneo, Gli occhi di Maria sulla Rivoluzione. «Miracoli» a Roma e nello Stato della Chiesa (1796-1797), Rome, Istituto nazionale di studi romani, 1995.

4. Notamment Sofia Boesch Gajano, «Dalla storiografia alla storia», Miracoli. Dai segni alla storia, éd. Ead. et Marilena Modica, Rome, Viella («Sacro/santo», nuova serie, 1), 1999, p. 215-233. 
peuple et aussi généralement du clergé. Cela ne veut évidemment pas dire que les miracles en question sont historiquement prouvés, chose du reste impossible, ni qu'ils n'ont pas été instrumentalisés par l'Église dans un but antifrançais et conservateur, au sens large du terme, mais simplement que la plus grande partie de ceux qui disaient voir les prodiges en était convaincue. Et c'est précisément cette adhésion massive qui constitue un problème historique et culturel qu'il faut examiner en évitant les raccourcis commodes, apologétiques ou de dénigrement ${ }^{5}$.

Selon Cattaneo, la peur des soldats français passant pour d'invincibles bêtes sanguinaires ramena à l'avant-plan une religiosité qui, malgré les attaques dont elles avaient fait l'objet au $\mathrm{XVIII}^{\mathrm{e}}$ siècle et les plaidoyers comme celui de Muratori en faveur d'une religion mieux ordonnée ${ }^{6}$, était restée vive et susceptible d'orienter les esprits et les regards. Encouragée et aussi canalisée par le clergé dans des circonstances difficiles, cette religiosité débordait largement le peuple pour toucher, de façon variable et avec de notables exceptions, toutes les couches de la société. Le besoin d'être rassuré par une intervention céleste, l'attente du miracle, l'effet d'entraînement suscité par la diffusion rapide de la nouvelle des prodiges expliquent cette vague miraculeuse accompagnée de manifestations de ferveur collective, qui elles-mêmes contribuaient au phénomène ${ }^{7}$.

Reste un point important pour notre propos sur lequel M. Cattaneo ne s'arrête pas: la délicate question du lien entre l'animation miraculeuse de l'image et son prototype céleste. À cet égard, un ouvrage de 1797, fort utilisé par Cattaneo pour d'autres aspects, contient quelques passages révélateurs. Il s'agit de la synthèse des procès canoniques relatifs aux images romaines faite par l'abbé Giovanni Marchetti, un célèbre polémiste antijanséniste et contre-révolutionnaire proche du pape Pie $\mathrm{VI}^{8}$. Le «ragionamento

5. Ibid., p. 79-80.

6. Cf. J.-M. Sansterre, «Les excès d'une dévotion mal réglée. L'utilisation des images religieuses et leur culte selon L. A. Muratori», Art et religion, éd. Alain Dierkens, Sylvie Peperstraete et Cécile Vanderpelen-Diagre, Bruxelles, Éditions de l'Université de Bruxelles («Problèmes d'histoire des religions », 20), 2010, p. 75-90.

7. M. Cattaneo, Gli occhi, p. 47-51 et passim.

8. Giovanni Marchetti, De' Prodigi avvenuti in molte sagre Immagini specialmente di Maria Santissima secondo gli autentici Processi compilati in Roma. Memorie Estratte e Ragionate [...] Con breve ragguaglio di altri simili Prodigi comprovati nelle Curie Vescovili dello Stato Pontificio, Rome, Zempel, 1797. Deux éditions, l'une avec gravures, l'autre sans; j'utilise l'édition avec gravures qui se trouve en ligne (Google books). 
preliminare» souligne qu'on ne saurait douter de la réalité de prodiges concernant tant d'images et dont tant de gens furent témoins ; tous ces gens ne sauraient avoir menti ni avoir été victimes d'une illusion. L'auteur explique exclusivement les miracles par la toute-puissance de Dieu et se montre fort prudent sur leur nature. Cela l'amène à proposer une alternative empruntée à la justification que saint Thomas d'Aquin donne des miracles eucharistiques ${ }^{9}$ :

Ou bien que Dieu ait opéré ces prodiges en provoquant un changement dans la matière figurée des visages sacrés, de sorte que tous voient cette variation miraculeuse, ou bien qu'il ait suscité cette sensation dans l'organe optique de chacun de ceux qui voyaient le prodige - ce sont les deux modes par lesquels, selon notre courte compréhension, il apparaît que le Très-Haut a pu se manifester dans son œuvre -, dans un cas ou dans l'autre, on comprend comment Dieu lui-même a pu retenir ou produire la miraculeuse impression ${ }^{10}$.

Dans le second cas, rien ne se passe dans l'image; dans les deux, elle reste passive et la Vierge elle-même n'intervient pas lors du miracle. Dieu, dit Marchetti un plus loin, a opéré des merveilles dans les images de sa très douce divine Mère - formulation qui répond au premier terme de l'alternative - pour qu'on l'invoque comme médiatrice avec une piété filiale et qu'on obtienne sa protection $^{11}$. À côté de ces données présentes dans l'introduction, les notices relatives aux diverses images montrent moins de réserve et l'on retrouve le flottement - plutôt que la contradiction - courant dans les récits hagiographiques lorsqu'il est question de l'auteur du miracle, tantôt attribué directement à Dieu, tantôt au saint. Ainsi lit-on que la Vierge «montre dans cette image qui est sienne le prodigieux mouvement des yeux $»^{12}$; qu'un marquis vit que

9. Thomas d'Aquin, Somme théologique, III ${ }^{\mathrm{a}}$ pars, Question 76, art. 8, cf. Jean-Pierre Delville, «Le "Sang de miracle" à la lumière de l'Originale de BoisSeigneur-Isaac : faits et interprétations », Le miracle du Saint Sang : Bois-SeigneurIsac 1405-2005, éd. Jean-Marie Cauchies et Marie-Astrid Collet-Lombard, Berlin, Lit («Vita regularis», 41), 2009, p. 43-117, ici p. 49. Je dois à J.-P. Delville d'avoir attiré mon attention sur cet emprunt qui m'avait échappé. Sans connaitre ce témoignage tardif, Megan Holmes, The Miraculous Image in Renaissance Florence, New Haven - Londres, Yale University Press, 2013, p. 172, considère que la justification donnée par saint Thomas pouvait permettre de comprendre les miracles où l'image est «transfigurée», mais il faudrait prouver qu'elle leur fut effectivement appliquée avant Marchetti.

10. G. Marchetti, De’ Prodigi, p. XLII-XLIII.

11. Ibid., p. XLVIII.

12. Ibid., p. 48. 
ses yeux très saints, comme s'ils avaient été animés (come se fossero animati), bougeaient transversalement d'un côté à l'autre comme pour regarder (quasi a mirare) avec amour tous les assistants et comme s'ils avaient voulu indiquer (come avesse voluto indicare) que la pieuse Mère avait plaisir aux louanges qu'on lui rendait et nous regardait tous d'un œil aimant ${ }^{13}$.

On aura remarqué l'insistance sur le come et quasi; la Vierge bouge les yeux comme si l'image s'animait et manifestait les sentiments de son prototype. Il y a là une certaine distanciation vis-à-vis de l'image «vivante» courante chez les clercs, mais dont il ne faudrait pas exagérer la portée. Lors de son interrogatoire canonique, le Père Gaetano Palma, par ailleurs postulateur de la cause de béatification de Benoît Joseph Labre, affirme:

dans certaines images, qu'à présent je ne puis plus individualiser avec précision, je distinguai, à l'occasion du susdit mouvement miraculeux, non seulement dans les yeux, mais encore sur le visage, quelque chose de vivace (un certo vivido) qui me semblait correspondre non à une peinture, mais bien à une personne vivante ${ }^{14}$.

C'est affirmer de façon mesurée et sans s'arrêter à une image particulière ce qui, pour Palma, était plus qu'une simple impression. Nous ne sommes pas très loin de ce que les gens simples devaient éprouver et croire. Lorsque, selon son témoignage, une femme du peuple dit à ses compagnes priant devant l'image de la Vierge dans la cathédrale d'Ancône: «Silence ! La Madone nous fait la grâce. Elle ouvre les yeux et elle rit», lorsqu'ensuite une gamine, allant voir de plus près, s'écrie «C'est vrai, c'est vrai, maman, la Madone ouvre les yeux et rit» et qu'enfin tous clament leur gratitude à la Reine du Ciel ${ }^{15}$, celle-ci apparaît comme agissant dans son image identifiée à elle sans qu'il y ait pour autant confusion entre le modèle et la représentation.

Quelques années plus tôt, à la fin de l'année 1789, une jeune actrice française, Louise Fusil, arriva à Bruxelles qui venait de se révolter contre le gouvernement autrichien. Dans ses Souvenirs qu'elle publia en 1841, elle raconte que la ville était en liesse «car la Vierge-Noire y faisait des miracles en faveur de la révolution [...]. Elle venait de manifester sa protection pour Vandernoot,

13. Ibid., p. 50.

14. Cité dans une autre perspective par M. Cattaneo, Gli occhi, p. 150.

15. Ibid., p. 209-210. 
le Lafayette du Brabant»- en fait, un avocat, figure de proue du courant conservateur de la Révolution brabançonne. On avait aperçu dans la main droite de la Vierge un papier que l'on supposa d'une grande importance. Un magistrat de la ville voulut le recevoir; la Vierge retira sa main, un membre du clergé n'eut pas plus de succès, mais «lorsqu'elle aperçut Vandernoot, elle avança gracieusement la main et lui remit ce papier, qui ne devait être confié qu'à lui et assurer le succès de son entreprise ». La Vierge se trouvait près de la ville, dans un endroit retiré. On l'amena en grande pompe à l'église métropolitaine. «Mais, dit Louise Fusil, il paraît que cette Vierge préférait l'air pur et le calme des champs; car, à la grande surprise des habitants, on la retrouva le lendemain dans son champêtre asile $»^{16}$.

Cette relation présente un contraste flagrant avec les témoignages relatifs à la vague miraculeuse de 1796/7 dans l'État pontifical: apparemment un seul récit sur un fait isolé raconté cinquante ans après le miracle, concernant une Vierge non identifiée - prétendue «noire» peut-être simplement parce qu'elle était miraculeuse -, un récit également qui n'est pas passé par le filtre d'autorités ecclésiastiques soucieuses de prouver l'authenticité des prodiges et de canaliser le phénomène. Il doit cependant refléter une rumeur, voire un essai plus concret d'instrumentalisation du miracle marial par un courant conservateur persuadé d'accomplir l'œuvre de la Providence et allant jusqu'à voir en Van der Noot un nouveau Moïse conduisant les enfants de Dieu au salut ${ }^{17}$. En l'occurrence, le vieux topos du retour miraculeux de l'image à son lieu de culte s'ajoute à une politisation du miracle marial envers son dévot. Le récit montre que l'animation prêtée aux images de la Vierge en cette période troublée de la fin du XVIII ${ }^{\mathrm{e}}$ siècle ne se limitait pas à l'Italie ${ }^{18}$.

16. Louise Fusil, Souvenir d'une actrice, édition critique par Valérie André, Paris, Honoré Champion («Bibliothèque des correspondances, mémoires et journaux », 26), 2006, p. 101-102. C'est Valérie André qui m'a fait connaître ce texte, je l'en remercie chaleureusement.

17. Pour le contexte politico-religieux, sans parler du récit de Fusil, voir Janet Polasky, «Providential History in Belgium at the End of the 18th Century», Revue belge de philologie et d'histoire, 55, 1977, p. 416-424 (référence communiquée par Jean-Jacques Heirweigh).

18. Pour la période contemporaine, que je n'ai pas à aborder ici, on verra à titre de comparaison l'ouvrage un peu trop engagé d'une ethnologue, Franca Romano, Madonne che piangono. Visioni e miracoli di fine millenio, Rome, Meltemi («Gli Argonauti », 24), 1997. 
UN PHÉNOMÈNE ENRACINÉ DANS LA LONGUE DURÉE: UNE LÉGENDE BYZANTINE ET L'ANIMATION D'IMAGES OCCIDENTALES JUSQU'AU MILIEU DU XIII ${ }^{\mathrm{E}}$ SIÈCLE

Il est temps, à présent, de remonter au Moyen Âge. Dans l'article récent publié en espagnol dont il a été question plus haut, je me suis efforcé de faire une synthèse de mes recherches sur les images «vivantes» avant le milieu du XIII ${ }^{\text {e }}$ siècle; $\mathrm{j}$ 'en reprends l'essentiel pour mon propos en y renvoyant pour de nombreuses autres données ${ }^{19}$. On parlera d'abord - car c'est indispensable pour ne pas fausser les perspectives - de l'animation des deux images du Christ, d'une part celle d'une légende byzantine qui, à partir des $\mathrm{X}^{\mathrm{e}}-\mathrm{XI}^{\mathrm{e}}$ siècles, connut une très large diffusion et un développement tout particulier en Occident, d'autre part un miracle romain de 921 rapporté à Saint-Gall que l'on peut considérer comme un des premiers, sinon le premier, événements de ce genre en Occident et un événement irréductible à tout modèle byzantin.

La légende d'origine byzantine, attestée à partir du VIII ${ }^{\mathrm{e}}$ siècle et attribuée erronément à saint Athanase d'Alexandrie (IV siècle), est celle de l'image du Christ de Beyrouth. Elle se répandit en Occident à partir du dernier tiers du $\mathrm{IX}^{\mathrm{e}}$ siècle. Elle raconte comment les juifs de Beyrouth réitérèrent les gestes prêtés à leurs ancêtres lors de la passion du Christ sur une image peinte du Sauveur. Quand ils lui percèrent le côté d'un coup de lance, du sang et de l'eau se mirent aussitôt à couler. Miracle qui prend tout son sens à la lecture de l'évangile de Jean où le jaillissement du sang et de l'eau, accomplissant l'Écriture, révèle à la fois la réalité du sacrifice et la divinité du Christ. L'eau et le sang sont considérés comme l'eau et le sang du Christ. Le récit montre qu'ils renouvellent les miracles christiques puisque les paralytiques, les aveugles et les possédés qui en ont été oints sont guéris. La légende eut un tel impact en Occident que l'on consacra pour la commémorer une fête liturgique à la Passion de l'image du Christ, fête déjà attestée au $\mathrm{x}^{\mathrm{e}}$ siècle à Limoges et à Gérone. Modèle consciemment utilisé ou à ce point intégré qu'il agissait sans qu'on s'y réfère, la légende constitua le substrat de quantité de miracles où les agresseurs de l'image - du

19. J.-M. Sansterre, «La imagen activada por su prototipo celestial» (voir n. 1), article qui lui-même repose en partie sur une série d'autres travaux dont certains seront cités ici. 
Christ ainsi que de la Vierge et d'autres saints - ne furent pas seulement des juifs, mais aussi des soudards impies, de mauvais chrétiens ayant perdu aux dés et, l'époque venue, des iconoclastes protestants, l'émission de sang prouvant la légitimité du culte des images ${ }^{20}$.

Ce type de miracle est bien connu. On sait moins que l'histoire du Christ de Beyrouth servit à justifier l'existence de reliques du saint sang. $\mathrm{Au} \mathrm{XI}^{\mathrm{e}}$ siècle, on ajouta au récit que l'évêque de Beyrouth reçut des juifs convertis le sang et l'eau divins et qu'il l'envoya dans des ampoules à de nombreuses églises de la chrétienté. L'addition n'est pas fortuite. On se demandait si à la résurrection, le Christ avait ou non repris le sang versé dans sa passion. Si l'on pensait qu'il l'avait repris, il fallait expliquer autrement l'existence des reliques du saint sang. On le fit en invoquant la passion de l'image et ce fut la solution retenue plus tard par saint Thomas d'Aquin qui, sans se référer explicitement à la légende, affirma que le saint sang provenait «d'une image du Christ que l'on avait percée $»^{21}$. Le sang passant pour provenir de l'image légendaire fut donc bien regardé comme le sang du Christ. Cela implique dans les croyances qu'au moment du miracle, le Christ a investi son image en revivant sa passion ou que, présent de façon latente en elle, il se manifeste

20. Id., «L'image blessée, l'image souffrante: quelques récits de miracles entre Orient et Occident $\left(\mathrm{VI}^{\mathrm{e}}-\mathrm{XII}^{\mathrm{e}}\right.$ siècle)», Les images dans les sociétés médiévales: pour une histoire comparée, éd. Id. et Jean-Claude Schmitt, Bruxelles-Rome, 1999 (= Bulletin de l'Institut historique belge de Rome, 69), p. 113-130, ici p. 116-122; Michele Bacci, "Quel bello miracolo onde si fa la festa del santo Salvatore": studio sulle metamorfosi di una leggenda», Santa Croce e Santo Volto. Contributi allo studio dell'origine e della fortuna del culto del Salvatore (secoli IX-XV), éd. Gabriella Rossetti, Pise, Gisem - ETS («Piccola Biblioteca Gisem», 17), 2002, p. 7-85; Carlo Espí Forcén, Recrucificando a Cristo. Los judíos de la Passio Imaginis en la isla de Mallorca, Mallorque, Objeto Perdido, 2009. Pour les joueurs de dés, voir les exemples donnés par Dominique Rigaux, Le Christ du dimanche. Histoire d'une image médiévale, Paris-Budapest-Turin, L'Harmattan («La librairie des humanités»), 2005, p. 176. Pour les iconoclastes protestants, voir notamment Olivier Christin, Une révolution symbolique. L'iconoclasme huguenot et la reconstruction catholique, Paris, Éditions de Minuit, 1991, p. 135-136, 242-243; Id., «Les miracles accomplis par l'intermédiaire des images ou les signes au secours des signes (1530-1630)», Les signes de Dieu aux XVI et XVII siècles, éd. Geneviève Demerson et Bernard Dompnier, Clermont-Ferrand, Faculté des Lettres et Sciences humaines de l'Université Blaise-Pascal (nouvelle série, 41), 1993, p. 79-87, surtout p. 84-87.

21. J.-M. Sansterre, «L'image blessée», p. 119-121; cf. Thomas d'Aquin, Somme théologique, $\mathrm{III}^{\mathrm{a}}$ pars, Question 54, art. 3. 
au moment clé de la nouvelle passion; d'une façon ou d'une autre, l'image s'identifie au moins le temps du miracle avec le prototype.

L'autre miracle christique se réfère à la Passion dans un contexte fort différent. Il est bien moins célèbre et, pour tout dire, il ne fait l'objet que d'une seule brève mention. Mais la nature de celle-ci permet de ranger résolument le miracle parmi les événements historiques, étant entendu, je le répète, que l'historien n'a pas à se prononcer sur la réalité même du prodige. La mention se trouve dans une brève continuation des annales alémaniques pour les années 921-926. Le continuateur, un moine de Saint-Gall, raconte sous l'année 921 que, le mercredi saint à Rome, près de l'autel de saint Pierre, la crucifixa imago Christi pleura en présence de tout le peuple au moment où on lisait la Passion. Les larmes tombant goutte à goutte formèrent comme des ruisseaux sur le pavement et au dos de la figure une sueur de sang se répandit jusqu'à terre ${ }^{22}$. La mention constitue un des trois faits que le continuateur relève pour les années en question, les deux autres étant une attaque hongroise contre son monastère et la mort du duc de Souabe. C'est dire l'importance attachée à l'événement. Il concerne une image réelle et typiquement occidentale, un grand crucifix. La figure du Crucifié prend part aux souffrances de son prototype non pas, comme dans la légende du Christ de Beyrouth, par le renouvellement des gestes impies de la Passion, mais lors de la commémoration liturgique de celle-ci, au moment d'une lecture déclamée qui exprimait dans ses modulations des sentiments intenses. Les souffrances du Crucifié se réactualisent par la conjonction de la lecture et de la présence monumentale du crucifix dans l'église, à une époque où cette présence constituait encore un fait remarquable puisque les premiers témoignages sur les crucifix remontent au siècle précédent. Loin d'être un phénomène tardif, l'animation miraculeuse de l'image majeure du christianisme occidental est proche de l'apparition de son culte. Bénéficiant du statut de la croix, les crucifix jouissaient d'une sacralité particulière et leur présence monumentale devait être fort impressionnante. La présence dans l'espace ecclésial de telles figures sacrées, en liaison avec un rituel collectif ou individuel ou

22. Éd. Georg Heinrich Pertz, Monumenta Germaniae Historica, Scriptores, t. I, Hannovre, 1826, p. 56; Walter Lendi, Untersuchungen zur frühalemannischen Annalistik. Die Murbacher Annalen, Fribourg (Suisse) («Scrinium Friburgense», 1), 1971, p. 192. 
du moins une attente des fidèles, amena fort tôt à les considérer parfois comme momentanément «vivantes ${ }^{23}$. Il est même question de leur prise de parole bien avant que, selon Thomas de Celano au milieu du XIII ${ }^{\mathrm{e}}$ siècle, le Christ du crucifix de San Damiano à Assise s'entretienne avec saint François ${ }^{24}$.

Une histoire, déjà attestée au $\mathrm{X}^{\mathrm{e}}$ siècle, représente un cas précoce d'activation d'une figure mariale. À Rome, la prestigieuse icône de Sainte-Marie in Tempulo, actuellement la Madonna di San Sisto, revint par la volonté de Dieu dans le monastère de moniales d'où l'avait enlevée le pape Serge III (904-911) pour la déposer au Latran, miracle sans doute lié à une revendication d'autonomie de la communauté de nonnes ${ }^{25}$. Ailleurs en Occident, il faut attendre plus longtemps pour voir s'animer des images de la Vierge peutêtre - en plus de la prépondérance du crucifix - parce qu'il était plus difficile d'admettre qu'un personnage céleste autre que l'auteur de tout miracle pût agir sur son image ${ }^{26}$. Il est significatif que ce fut d'abord à l'Enfant d'une sedes sapientiae qu'on attribua la parole. Vers 1080, Goscelin de Saint-Bertin, un bénédictin flamand établi en Angleterre, raconte déjà une histoire qui deviendra un célèbre exemplum. À Spire, une «image de la sainte Mère de Dieu avec son enfant» était vénérée à une place si humble que les enfants pouvaient la toucher. Un petit garçon offre un morceau de pain à l'enfant Dieu et, comme la sacra imago ne bouge pas, il l'embrasse en répétant «Puppe, pappa; puppe, pappa» («petit garçon, mange»). Finalement «l'image de l'Enfant tout-puissant»l'étreint à son tour et lui dit: «Pupe, dans trois jours tu mangeras (pappabis)

23. Cf. J.-M. Sansterre, «Visions et miracles en relation avec le crucifix dans des récits des $\mathrm{X}^{\mathrm{e}}-\mathrm{XI}^{\mathrm{e}}$ siècles», Il Volto Santo in Europa. Culto e immagini del Crocifisso nel Medioevo, éd. Michele Camillo Ferrari et Andreas Meyer, Lucques, Istituto storico lucchese, 2005, p. 387-406, ici p. 389-390 et passim pour d'autres cas; Id., «La imagen activada por su prototipo», p. 79-87.

24. Ibid., p. 84-85 (avec les travaux indiqués).

25. La plus ancienne version, découverte par Guy Philippart dans un manuscrit conservé à Saint-Gall, est étudiée et éditée dans un mémoire de licence de l'Université catholique de Louvain effectué sous la direction de J. Pycke et G. Philippart: Julie de Peñaranda de Franchimont, Serge III et le miracle de l'icône mariale de Santa Maria in Tempulo, année académique 2005-2006 [dactyl.]. Une version du $\mathrm{XI}^{\mathrm{e}}$ siècle est éditée et commentée par Gerhard Wolf, Salus Populi Romani. Die Geschichte römischer Kultbilder im Mittelalter, Weinheim, VCH Acta humaniora, 1990, p. 162-166 et 318-320 (Quellen n ${ }^{\circ}$ ).

26. Je développe cette hypothèse dans «La imagen activada por su prototipo», p. 87-90. 
avec moi», ce que la mère du petit garçon entendit avec effroi. Trois jours plus tard, l'enfant mourut et devint le convive du Christ au ciel ${ }^{27}$. Le geste et la parole du petit ont incité le Christ à investir son image en adoptant, dans sa condescendance, le langage enfantin (puppe, pappabis). On trouve déjà là, de façon originale, un trait relevé brièvement par Olivier Christin, «l'antériorité fréquente de la parole humaine qui sollicite, appelle, désire et au fond éveille la pierre ou le bois dont l'intercesseur divin [j'ajoute: ou Dieu luimême] va se servir pour lui répondre $»^{28}$.

Le premier témoignage que je connaisse d'une animation miraculeuse de la Vierge elle-même en Occident au-delà de l'Italie figure dans l'autobiographie de Guibert de Nogent écrite en 1114-1115. Guibert relate un fait survenu dans le dernier tiers du XI ${ }^{\mathrm{e}}$ siècle alors qu'il était moine à Saint-Germer-de-Fly en Picardie. Un 18 juin au matin, la foudre tomba dans l'église abbatiale, brisa un grand crucifix, dont la tête et le bras droit furent détruits et tua deux moines, laissant les autres dans la stupeur. Peu après, Guibert vit «l'image de la bienheureuse Mère de Dieu, qui se dressait en dessous du crucifix, avec un visage si agité, si éloigné de sa sérénité habituelle qu'il apparaissait totalement autre». Guibert ne voulut pas en croire ses yeux avant de savoir que d'autres avaient observé la même chose. Les moines finirent par comprendre qu'ils avaient mérité un juste châtiment pour leurs péchés et ils virent aussitôt le visage de la Vierge retrouver sa sérénité2 ${ }^{2}$. La destruction du grand crucifix manifestement délaissé par son prototype focalisa l'attention des

27. Goscelin de Saint-Bertin, Liber confortarius, éd. C. H. Talbot, dans Analecta monastica. Textes et études sur la vie des moines au Moyen Âge, $3^{\mathrm{e}}$ série, éd. Id., M. Lebreton et J.-C. Leclercq, Rome, Herder («Studia Anselmiana», 37), 1955, p. 107-108. Pour les mentions postérieures: Karin Fuchs, «Les collections de Miracles de la Vierge: rassembler, copier, réécrire. L'exemple du récit du pain offert à l'image du Christ», Miracles, Vies et réécritures dans l'Occident médiéval, dir. Monique Goullet et Martin Heinzelmann, Ostfildern, Thorbecke («Beihefte der Francia », 65), 2006, p. 67-89.

28. Olivier Christin, «Faire parler les statues. Expériences de l'époque moderne», dans René Wetzel et Fabrice Flückiger, Die Predigt im Mittelalter zwischen Mündlichkeit, Bildlichkeit, und Schriftlichkeit. La prédication orale au Moyen Âge entre oralité, visualité et écriture, Zürich, Chronos ( MedienwandelMedienwechsel-Medienwissen», 13), 2010, p. 329-346, ici p. 333.

29. Guibert de Nogent, De vita sua (Autobiographie), I, 23, éd. et trad. Edmond-René Labande, Paris, Les Belles Lettres («Les classiques de l'histoire de France au Moyen Âge»), 1981, p. 178-183, surtout p. 182. 
moines stupéfaits sur l'image mariale qu'ils perçurent comme réagissant à l'événement.

Les mentions concernant la Vierge augmentent de façon significative dans le courant du XII ${ }^{\mathrm{e}}$ siècle et plus encore au siècle suivant, ce qui coïncide avec le plein épanouissement de la ferveur mariale au Moyen Âge. Elles figurent surtout dans nombre de récits édifiants, souvent peu contextualisés, où l'on voit des statues de la Vierge bouger et parler pour intercéder, protéger, récompenser, ramener sur le droit chemin ou punir ${ }^{30}$. Certains récits ont récemment fait l'objet d'une lecture «genrée» fort séduisante de Katherin Allen Smith. La beauté physique des statues de Marie, une beauté perçue par les hommes comme hautement féminisée, leur donnait une corporéité qui contribua sans doute à leur reconnaître des manifestations de vie ${ }^{31}$. Cette lecture trouve une de ses meilleures illustrations dans une histoire fort répandue: une statue de la Vierge mise devant une église en reconstruction replie le doigt sur l'anneau qu'un tout jeune homme, jouant à la pelote devant le portail, lui avait passé en promettant de servir sa vie entière une femme aussi belle; plus tard, la Vierge le contraignit à tenir son engagement ${ }^{32}$.

Une histoire que le cistercien allemand Césaire de Heisterbach raconte vers 1220 dans un célèbre recueil de miracles rédigé pour les novices de son monastère - une histoire raillée plus tard par la critique protestante ${ }^{33}$ - illustre de façon spectaculaire le pouvoir d'intercession de la Vierge. Un chevalier avait renié son Créateur sans se résoudre à renier Marie. Entrant dans une église, il se mit à éprouver du repentir devant la statue de Marie avec l'Enfant placée sur l'autel et à implorer la Vierge. «Par la bouche de son image», l'avocate des chrétiens demande à son fils d'avoir pitié; l'Enfant se détourna; l'image se leva, posa l'Enfant sur l'autel et se prosterna

30. Pour de multiples exemples, cf. J.-M. Sansterre, «La Vierge Marie et ses images chez Gautier de Coinci et Césaire de Heisterbach », Viator, 41, Multilingual, 2010, p. 147-178.

31. Katherine Allen Smith, «Bodies of Unsurpassed Beauty: "Living" Images of the Virgin in the High Middle Ages », Viator, 37, 2006, p. 167-187.

32. Bibliographie sur ce récit: J-M. Sansterre, «La Vierge Marie et ses images », p. 156, n. 51.

33. Cf. Id., «Les images parlantes des catholiques, du Moyen Âge aux Temps modernes, et la polémique protestante $\left(\mathrm{XVI}^{\mathrm{e}}-\mathrm{XVII}^{\mathrm{e}}\right.$ siècles). Une première approche», à paraître dans Faire parler et faire taire les statues de l'Antiquité à nos jours, dir. Caroline Michel d'Anoville et Yann Rivière ( Collection de l'École française de Rome»), n. 19 et texte correspondant. 
à ses pieds en réitérant sa demande; l'Enfant releva sa mère et lui accorda ce qu'elle souhaitait ${ }^{34}$. Conscient de l'étrangeté des miracles de ce genre et sans doute du scepticisme de certains - la croyance en ces prodiges n'était pas unanimement partagée, ni parmi les clercs, ni parmi les laïcs ${ }^{35}$-, Césaire cherche à les justifier de façon explicite, ce qui est très rare. On trouve dans cette justification le flottement courant sur l'auteur des miracles que nous avons observé plus haut chez Marchetti. Césaire note d'abord que «les saints opèrent de nombreux miracles dans et par leurs images, surtout dans les lieux où elles sont vénérées», puis il attribue les miracles à l'Esprit divin qui anime la matière ${ }^{36}$. Cette solution plus sûre sur le plan théologique ne rend pas compte de la croyance sous-jacente à l'animation miraculeuse, ce que condense en revanche la formule sancti in et per suas imagines virtutes operantur. Le prototype agit de l'extérieur sur l'image, ou bien il l'investit temporairement ou encore il y est présent de façon latente. Fort révélateur aussi, car il émane d'un membre de l'élite cléricale s'adressant à ses confrères, un souvenir relaté peu après 1263 par l'ancien maître général des dominicains, Humbert de Romans, pour montrer que la Vierge était la «patronne particulière» de son ordre. Au temps de son noviciat, quelque quarante ans plus tôt, on lui avait rapporté qu'une sainte recluse, voyant des jeunes dominicains courir par le monde, s'était demandé devant une image de la Vierge s'ils pourraient se garder des péchés du monde. Alors «elle entendit une voix comme (quasi) de l'image lui dire: "N'aie crainte pour ces frères, parce que je les ai reçus sous ma protection" ${ }^{37}$. Le quasi, utilisé pour marquer la différence entre le modèle et sa représentation, ne fait guère illusion. C'est bel et bien d'un miracle d'image parlante dont il est question.

Dans un cas, la présence du prototype va jusqu'à sa partielle incarnation dans l'image en modifiant pour toujours sa nature

34. Césaire de Heisterbach, Dialogus Miraculorum, II, 12, éd. Joseph Strange, 2 vols., Cologne - Bonn - Bruxelles, 1851, vol. I, p. 78-81.

35. J.-M. Sansterre, «La Vierge Marie et ses images», p. 177, avec une citation de Guy Philippart.

36. Césaire de Heisterbach, Dialogus Miraculorum, VII, 45, vol. 2, p. 64 ; cf. J.-M. Sansterre, op. cit., p. 169-174.

37. Humbert de Romans, Expositio super constitutiones fratrum praedicatorum, éd. Joachim Joseph Berthier, B. Humberti de Romanis opera de vita regulari, 2 vols., Rome, Marietti, 1956, vol. 2, p. 136. 
puisque la matière même est définitivement transformée. Or, contrairement à beaucoup de récits que je viens d'évoquer, il s'agit d'une image miraculeuse individualisée et réellement vénérée dans un lieu déterminé: Notre-Dame de Sardenai, une icône attestée à partir du XII ${ }^{\mathrm{e}}$ siècle et encore vénérée aujourd'hui au même endroit, le monastère de Saydnaya à 22 kilomètres au nord-est de Damas. On racontait que ses seins étaient devenus et restés chair et l'on recueillait l'huile miraculeuse qui en émanait. La légende et l'huile relique se répandirent en Occident vers la fin du XII ${ }^{\mathrm{e}}$ siècle $^{38}$.

En Occident même, avant la seconde moitié du XIII ${ }^{\mathrm{e}}$ siècle, les récits miraculaires locaux ne mentionnent pas beaucoup d'images miraculeuses de la Vierge, mais il y a de notables exceptions et, parmi elles, des images «vivantes». En 1187, lors d'une guerre entre les rois de France et d'Angleterre, eut lieu un double miracle christique et marial, rapporté de façon concordante par des sources anglaises et françaises de l'époque. À Déols près de Châteauroux dans le Berry, un mercenaire à la solde du roi d'Angleterre lança une pierre contre une statue de la Vierge à l'Enfant placée au-dessus du porche de l'église abbatiale. La pierre brisa le bras de l'Enfant; un flot de sang divin, explicitement comparé à celui de l'image de Beyrouth, se répandit sur le sol et le sacrilège mourut aussitôt. Le lendemain soir, les fidèles virent l'effigie de la Vierge se mouvoir comme si elle voulait se transporter ailleurs, et, saisissant des mains les deux extrémités de son vêtement - un «vêtement de pierre», insiste une source -, elle le déchira et dénuda sa poitrine. L'image s'identifie à son prototype, elle devient la Mère compatiens de la dévotion mariale du $\mathrm{XII}^{\mathrm{e}}$ siècle; elle agit, selon une source anglaise, «comme si elle prenait part aux souffrances de son fils». Ce double miracle frappa les esprits au point d'être directement ou non la cause d'une trêve entre les deux rois. «Des deux côtés, observe un chroniqueur contemporain, les chevaliers en armes étaient terrifiés

38. Entre autres, Bernard Hamilton, «Our Lady of Saidnaiya: An Orthodox Shrine Revered by Muslims and Knights Templar at the Time of the Crusades », The Holy Land, Holy Lands, and Christian History, éd. Robert Norman Swanson, Woodbridge, Published for the Ecclesiastical History Society by the Boydell Press («Studies in Church History», 36), 2000, p. 207-215; Michele Bacci, «A Sacred Space for a Holy Icon: The Shrine of Our Lady of Saydnaya», Hierotopy. The Creation of Sacred Spaces in Byzantium and Medieval Russia, éd. Alexei Lidov, Moscou, Indrik, 2006, p. 373-387. 
et de très nombreux doutaient de l'issue de la guerre ${ }^{39}$. Quelques années plus tard, vers 1196, avant une guerre entre Alphonse IX de León et Alphonse VIII de Castille, une image de la Vierge avec l'Enfant se mit à saigner à Saint-Étienne, hors les murs de León. La statue fut alors portée en procession à Saint-Isidore et posée sur le maître-autel, où elle versa continuellement du sang pendant trois jours. C'était un présage de l'horrible guerre et de pertes pour SaintIsidore $^{40}$.

\section{LA PROLIFÉRATION DES IMAGES «VIVANTES» (SECONDE MOITIÉ DU XIII ${ }^{\mathrm{E}}-\mathrm{XVII}{ }^{\mathrm{E}}$ SIÈCLE)}

Dans les derniers siècles du Moyen Âge, les miracles d'images animées se multiplient à foison tant dans les expériences mystiques que sous une forme publique et collective. On a maintes fois souligné que les expériences mystiques avaient souvent lieu devant des images matérielles et que les images mentales dépendaient largement de celles-ci ${ }^{41}$. Cela concernait avant tout les effigies du

39. Jean Hubert, «Le miracle de Déols et la trêve conclue en 1187 entre les rois de France et d'Angleterre», Bibliothèque de l'École des Chartes, 96, 1935, p. 285-300; André Vauchez, «L'image vivante: quelques réflexions sur les fonctions des représentations iconographiques dans le domaine religieux en Occident aux derniers siècles du Moyen Âge », Pauvres et riches. Société et culture du Moyen-Âge aux Temps Modernes. Mélanges offerts à Bronislaw Geremek, Varsovie, Wydawnictno naukowe PWN, 1992, p. 231-240, ici p. 236, repris sous le titre «Les images saintes: représentations iconographiques et manifestations du sacré», dans Id., Saints, prophètes et visionnaires. Le pouvoir surnaturel au Moyen Âge, Paris, Albin Michel («Histoire»), 1999, p. 79-91, 240-242, ici p. 85-86; J.-M. Sansterre, «Omnes qui coram hac imagine genua flexerint... La vénération d'images de saints et de la Vierge d'après les textes écrits en Angleterre du milieu du $\mathrm{XI}^{\mathrm{e}}$ aux premières décennies du $\mathrm{XIII}^{\mathrm{e}}$ siècle», Cahiers de civilisation médiévale, 49, 2006, p. 257-294, ici p. 278-280 où l'on trouvera les passages cités.

40. Lucas de Tuy, Chronicon mundi, IV, 84, éd. Emma Falque, Turnhout, Brepols («Corpus Christianorum, Continuatio medievalis», 74), 2003, p. 323 ; cf. J.-M. Sansterre et Patrick Henriet, «De l'inanimis imago à l'omagem mui bella. Méfiance à l'égard des images et essor de leur culte dans l'Espagne médiévale ( $\mathrm{VII}^{\mathrm{e}}-\mathrm{XIII}{ }^{\mathrm{e}}$ siècle)», Edad Media. Revista de Historia, 10, 2009, p. 37-92, ici. p. 79; Francesca Español Bertran, «El milagro y su instrumento icónico. La fortuna de las imágenes sagradas en el ámbito peninsular», Codex Aquilarensis, 29, 2013, p. 117-134, ici p. 119.

41. Entre autres, Chiara Frugoni, «Le mistiche, le visioni e l'iconografia: Rapporti e influssi», Temi e problemi nella mistica femminile trecentesca, Todi, 1983 («Convegni del Centro di studi sulla spiritualità medievale», 20), 1983, p. 5-45; Jeffrey F. Hamburger, Nuns as Artists. The Visual Culture of a Medieval 
Christ comme c'était déjà le cas aux $\mathrm{XII}^{\mathrm{e}}$-XIII ${ }^{\mathrm{e}}$ siècles. Parmi les témoignages relatifs à la Vierge, je retiens un curieux passage de la Vie, écrite entre 1317 et 1329 , de sainte Kinga appelée aussi Cunégonde, une princesse hongroise du XIII ${ }^{\mathrm{e}}$ siècle fondatrice d'un couvent de clarisses où elle vécut après la mort de son époux. Le passage concerne ce que vit et entendit une sœur qui était entrée dans l'oratoire de Kinga alors que celle-ci était en pleine contemplation:

elle la vit en larmes et parlant à une image de la glorieuse vierge Marie et elle entendit une voix venant de l'image, une voix qu'elle ne comprenait absolument pas, qui s'avançait vers l'heureuse dame dans quelque chose de très brillant et elle vit la dame resplendir d'une clarté toute spéciale ${ }^{42}$.

Cette description de sons incompréhensibles de l'extérieur arrivant à leur destinataire dans un rayon lumineux est exceptionnelle; elle s'inspire peut-être des phylactères portant des paroles dans les peintures ${ }^{43}$.

Convent, Berkeley - Los Angeles - Londres, University of California Press, 1997, trad. Catherine Bédard et Daniel Arasse, Peindre au couvent. La culture visuelle d'un couvent médiéval, Paris, Gérard Monfort («Imago mundi»), 2000; Id., The Visual and the Visionary. Art and Female Spirituality in Late Medieval Germany, New York, Zone Books, 1998; Peter Dinzelbacher, «Religiöses Erleben vor bildender Kunst in autobiographischen und biographischen Zeugnissen des Hochund Spätmittelalters», Frömmigkeit im Mittelalter. Politisch-soziale Kontexte, visuelle Praxis, körperliche Ausdrucksformen, éd. Klaus Schreiner et Marc Müntz, Munich, Fink, 2002, p. 299-330; Bruno Boerner, «Le rôle de l'image sculptée dans les couvents féminins allemands à la fin du Moyen Âge», Bibliothèque de l'École des chartes, 162, 2004, p. 119-131; Katherine L. Jansen, «Miraculous Crucifixes in Late Medieval Italy ", Signs, Wonders, Miracles. Representations of Divine Power in the Life of the Church, éd. Kate Cooper et Jeremy Gregory, Woodbridge, Published for the Ecclesiastical History Society by the Boydell Press ( Studies in Church History», 41), 2005, p. 203-227; Barbara Newman, «The Visionary Texts and Visual Worlds of Religious Women», Crown and Veil. Female Monasticism from the Fifth to the Fifteenth Centuries, éd. Jeffrey Hamburger et Susan Marti, New York, Columbia University Press, 2008, p. 151-171; Éric Palazzo, «Visions and Liturgical Experience in the Early Middle Ages», Looking Beyond. Visions, Dreams, and Insights in Medieval Art \& History, éd. Colum Hourihane, Princeton (N.J.), Index of Christian Art Princeton University in association with Penn State University Press («Index of Christian Art. Occasional Papers», 11), 2010, p. 15-29; Jacqueline E. Jung, «The Tactile and the Visionary: Notes on the Place of Sculpture in the Medieval Religious Imagination », ibid., p. 203-240.

42. Vita et miracula sanctae Kyngae ducissae Cracoviensis, 32, éd. Wojciech Ketrzynki, dans Monumenta Poloniae Historica, t. IV, Lwów, p. 709.

43. Sur les paroles figurées, voir en particulier Cécile Voyer, « Voir et entendre: des paroles dans l'image. À propos d'une enluminure du manuscrit 121 de la Bibliothèque municipale d'Avignon », La Parole sacrée. Formes, fonctions, sens $\left(X I^{e}-X V^{e}\right.$ siècle), Toulouse, Privat («Cahiers de Fanjeaux», 47), 2013, p. 387-406. 
Les expériences visionnaires ne restèrent pas limitées à des mystiques de haut vol. Leurs mentions sont tellement fréquentes dans les livres des sœurs dominicaines de l'espace allemand de la fin du XIII ${ }^{\mathrm{e}}$ et des $\mathrm{XIV}^{\mathrm{e}}-\mathrm{XV}^{\mathrm{e}}$ siècles que Barbara Newman a pu parler à leur propos de «démocratisation radicale de la grâce». Elle précise: «Des phénomènes qui autrefois caractérisaient seulement des saints - visions, voix, clairvoyance, multiplication de nourriture, lévitation même - faisaient maintenant partie du quotidien de la communauté ${ }^{44}$. Tous les récits ne traduisent pas cependant des expériences réelles; ils pouvaient aussi, comme l'observe Jeffrey Hamburger, viser à structurer la dévotion, à guider l'imagination des nonnes à partir de la prière devant les images ${ }^{45}$.

Je m'arrêterai davantage à ce qui relève plutôt du culte public des images miraculeuses - bien qu' il n'y ait pas de clivage avec la dévotion privée. À partir de la deuxième moitié du XIIII et plus encore du $\mathrm{XIV}^{\mathrm{e}}$ siècle, on voit se multiplier les mentions d'images miraculeuses mariales attachées à un lieu déterminé qu'elles sacralisent ou contribuent à sacraliser et qui leur donne ou contribue à leur donner une forte identité. On peut rejoindre ici Caroline Bynum et inscrire l'évolution dans le phénomène plus large de l'importance croissante attachée aux objets matériels comme lieux et révélateurs du divin ${ }^{46}$, ou, comme le dit André Vauchez, de l'accent mis par l'Église «sur tous les signes visibles et tangibles de la présence de Dieu au milieu des hommes ${ }^{47}$. Bien qu'on manque encore d'une étude globale - ce qui, il est vrai, tient de la gageure -, nombre de travaux de qualité ont montré l'importance prise par le culte des images miraculeuses en divers endroits à la fin du Moyen Âge et à l'époque moderne ${ }^{48}$.

44. B. Newman, «The Visionary Texts», p. 163.

45. J. F. Hamburger, Nuns as Artists, p. 94-95; trad. Peindre au couvent, p. 62-65.

46. C. W. Bynum, Christian Materiality (voir n. 1), relève ce qu'elle considère comme un paradoxe caractéristique de la religiosité des derniers siècles du Moyen Âge: à la fois l'accroissement de l'importance attachée à la matière comme «locus» du sacré et l'essor d'une intériorisation de la piété rejetant le pouvoir de la matière. Sans perdre de vue le second pôle du paradoxe, l'intériorité, Bynum consacre son livre au premier, la «matérialité chrétienne», celle des images, des reliques, des sacramentaux et bien sûr des espèces eucharistiques.

47. A. Vauchez, «L'image vivante», p. $238=$ Saints, prophètes et visionnaires, p. 88 .

48. Les études se sont multiplées depuis les articles pionniers d'A. Vauchez, «L'image vivante» (voir n. 39) et, avant lui, de Richard C. Trexler, «Florentine Religious Experience: the Sacred Image», Studies in the Renaissance, 19, 1972, 
La plupart mettent en évidence, à des degrés divers, l'interaction multiforme entre les images, leurs spectateurs et surtout leurs dévots conduisant à ce qui était vécu et raconté comme des miracles ${ }^{49}$. L'animation des statues ou des peintures y figurent en bonne place, si bien qu'en 1582 le cardinal Gabriele Paleotti l'inclut dans les critères de sa définition des images sacrées. Parmi celles-ci, il y a les images dans lesquelles et par lesquelles Dieu opère des miracles: elles se déplacent en divers endroits, leur face se met à resplendir, elles pleurent, elles saignent, elles font quelque mouvement d'une personne comme si elles étaient vivantes, ou encore la divine bonté

p. 7-41, réimpr. Id., Church and Community 1200-1600. Studies in the History of Florence and New Spain, Rome, Storia e Litteratura («Raccolta di studi e testi», 168), 1987, p. 37-74. Parmi les travaux récents, on verra notamment Michele Bacci, «Pro remedio animae». Immagini sacre e pratiche devozionali in Italia centrale (secoli XIII e XIV), Pise, Gisem - Edizioni ETS («Piccola Biblioteca Gisem», 15), 2000; The Miraculous Image in the Late Middle Ages and Renaissance, éd. Erik Thun $\varnothing$ et Gerhard Wolf, Rome, «L'Erma» di Bretschneider, 2004 - sur un plan général, une belle introduction d'André Vauchez (ibid., p. 9-14) et une non moins importante contribution de Richard C. Trexler, «Being and NonBeing. Parameters of the Miraculous in the Traditional Religious Image», ibid., p. 15-27; William A. Christian jr., "Images as Being in Early Modern Spain», Sacred Spain. Art and Belief in the Spanish World, éd. Ronda Kasl, Indianapolis Museum of Art, distribued by Yale University Press, 2009, p. 75-119; Annick Delfosse, La «Protectrice du Païs-Bas». Stratégies politiques et figures de la Vierge dans les Pays-Bas espagnols, Turnhout, Brepols ( EÉglise, liturgie et société dans l'Europe moderne», 2), 2009, passim; M. Holmes, The Miraculous Image in Renaissance Florence (voir n. 9); Jane Garnett et Gervase Rosser, Spectacular Miracles. Transforming Images in Italy from the Renaissance to the Present, Londres, Reaktion Books, 2013. Pour les miracles, cf. aussi: François Lecercle, Le signe et la relique. Les théologies de l'image à la Renaissance, thèse pour le doctorat d'État, Université Paul-Valéry (Montpellier III), 1987 [non publiée, mais accessible en prêt], vol. II, p. 509-556 (chap. XII, «Infigurabilité de l'événement. Les miracles de l'image»); O. Christin, «Les miracles accomplis par l'intermédiaire des images» (voir n. 20); Paola Vismara Chiappa, Miracoli settecenteschi in Lombardia tra istituzione ecclesiastica e religione popolare, Milan, Istituto propaganda libreria («Il sestante»), 1988, surtout p. 9-44; Maurizio Sangalli, Miracoli a Milano. I processi informativi per eventi miracolosi nel Milanese in età spagnola, Milan, NED («Archivio Ambrosiano», 67), 1993, passim; Guilio Sodano, Il miracolo nel Mezzogiorno d'Italia dell'età moderna tra Santi, Madonne, guaritrici e medici, Naples, Guida ("Passagi e percorsi,» 21. «Saggi», XV), 2010, surtout p. 149-215. Pour la période contemporaine, un livre indispensable: Marlène Albert Llorca, Les Vierges miraculeuses. Légendes et rituels, Paris, Gallimard, 2002 («Le temps des images»), 2002, remarquable étude anthropologique sensible à la dimension historique, centrée sur les Vierges catalanes. Voir également infra n. 58, 60.

49. On verra en particulier J. Garnett et G. Rosser, Spectacular Miracles, qui placent de façon significative cette interaction au centre de leur recherche. 
opère des guérisons par elles ${ }^{50}$. Il va sans dire que dans un traité de ce genre, écrit de surcroît moins de vingt ans après le concile de Trente, les miracles d'image ne pouvaient être attribués qu'à Dieu seul.

Ce type de miracles constituait une cible de choix pour la polémique protestante qui déniait toute faculté de vie aux images dont le culte était taxé de superstition et d'idolâtrie. «Vostre image est une souche en bois / Sans entendement, ne vertu; / elle est semblable à un festu / qui ne se peut remuer», disaient en 1563 les huguenots à propos de «l'idole» de Nostre-Dame du Puy ${ }^{51}$. Féroce satiriste, le calviniste Philippe de Marnix de Sainte-Aldegonde ( $† 1598)$ raille la profusion des prodiges en termes crus que je laisse dans l'original:

Il ny a chapelle si malotrue qui ne se piaffe d'avoir quelque extraordinaire et miraclifique image de nostre Seigneur ou de nostre Dame, ou de sa mere saincte Anne, ou de saincte Barbe, ou de saincte Marguerite, ou de quelque autre sainct ou saincte, laquelle (...) ait quelque fois ou parlé, ou ris, ou pleuré, ou sué, ou petté, ou vessé, ou fait un niquet de la teste, ou un sonnet du cul, ou en somme ait donné quelque preuve de sa vaillantise. Nos legendes en sont pleines comme un chien de pulces ${ }^{52}$.

Pourtant, du côté catholique, ces miracles faisaient l'objet d'une vigilance croissante en raison des fréquentes supercheries ${ }^{53}$,

50. Gabriele Paleotti, Discorso intorno alle imagini sacre et profane, I, 16, éd. Paola Barocchi, Trattati d'arte del Cinquecento fra manierismo e controriforma, vol. II, Bari, Laterza («Scrittori d'Italia», 221), 1961, p. 198; cf. M. Holmes, The Miraculous Image, p. 6-7.

51. Avertissement à Messieurs du Puy, touchant l'idolatrie qu'ils commettent envers l'idole de leur Nostre-Dame, cité par Alain Joblin, «Les protestants, Marie et le culte marial», La dévotion mariale de l'an mil à nos jours, dir. Bruno Béthouard et Alain Lottin, Arras, Artois Presse Université («Histoire»), 2005, p. 323-336, ici p. 333. Pour un aspect de la polémique, celui concernant les images parlantes, cf. l'article mentionné supra $\mathrm{n} .33$.

52. Philippe de Marnix de Sainte Aldegonde, Tableau des différends de la religion, éd. Edgar Quinet (reprenant le texte de l'éd. de Leyde, 1601), 4 vols., Bruxelles, Van Meenen, 1857, vol. II, p. 367. Marnix poursuit en s'amusant des dires et des exempla de Césaire de Heisterbach et il ajoute: «Mais qui ne sçait que toutes nos Eglises, voire toutes les rues, carrefours et coings de nos maisons, regorgent de semblables images? Et les miracles qu'elles font en sont drus et menus comme la neige sur le mont Cenis, ou comme le sable aux deserts de Lybie» (ibid.).

53. Un cas célèbre, qui donna lieu à de retentissants procès et valut le bûcher aux responsables, est celui de quatre religieux du couvent dominicain de Berne qui, en 1507, firent parler la Vierge d'une Pietà et lui firent verser des larmes de sang. Je me limite ici à renvoyer à Franz-Josef Sladeczek, «L'affaire Jetzer», Iconoclasme. Vie et mort de l'image médiévale. Catalogue de l'exposition Musée d'histoire de Berne et Musée de l'œuvre Notre-Dame, Musées de Strasbourg, dir. Cécile Dupeux, Peter Jezler et Jean Wirth, Paris, Somogy, 2001, p. 254-255. 
causes de scandale pour les fidèles et aliments de polémique pour les adversaires. Ils pouvaient même être carrément rejetés. Dans un sermon de carême prononcé à Padoue en 1455, le célèbre prédicateur franciscain Robert de Lecce n'hésite pas à s'y opposer de façon catégorique:

Plusieurs disent qu'une image pleure; je n'ai jamais cru cela et tu ne dois pas le croire, car ce sont des balivernes. Je me souviens que, dans la cité où je suis né, il y avait dans la maison d'un particulier une image de la Vierge avec du vernix liquide. Le bonhomme alluma une grande torche et la posa près de l'image; en raison de la chaleur, la poix de cire se liquéfia et l'homme se mit à crier 'Miséricorde'. La cité accourut. L'évêque, apprenant cela, ordonna au peuple de ne pas se rendre en cet endroit et, la vérité découverte, il fit mettre en prison le bonhomme et ses complices. Ensuite l'image ne pleura plus. C'est une sottise de croire qu'il y aurait quelque chose de divin dans une image ${ }^{54}$.

Sans aller jusque-là, d'autres se montraient inquiets des risques d'illusion. Geert Groote ( $† 1384)$, l'initiateur de la devotio moderna, observait déjà :

Un homme simple croit sentir avec ses sens extérieurs la présence du Christ ou d'un saint dont il n'a que l'image; il le voit avec ses yeux, l'entend de ses oreilles, le touche de ses mains. Et cette illusion n'est pas sans danger, car ces gens prennent les signes pour des choses, comme si quelqu'un prenait l'image du Christ pour le Christ ${ }^{55}$.

Ce qui était pour lui «les fruits d'une imagination échauffée par la dévotion $»^{56}$ devient deux siècles plus tard tromperie du démon pour Jean de la Croix, qui ne peut toutefois pas nier la possibilité du miracle, ni même sa fréquence:

Il arrive que, regardant une image, on la voit bouger ou changer d'expression, faire comprendre des choses ou parler. Bien que souvent ce genre d'effets surnaturels provenant des images soit bon et véritable

54. Robert de Lecce, De adoratione Dei, 33, éd. Oriana Visani, Roberto da Lecce. Quaresimale Padovano 1455, Padoue, Messaggero ( «Centro studi antoniani», 6) = Il Santo, ser. II, 23, fasc. 1-2, gennaio - agosto 1983, p. 139.

55. Geert Groote, De quattuor generibus meditabilium, trad. Georgette ÉpineyBurgard, Gérard Grote fondateur de la Dévotion Moderne. Lettres et traités, Turnhout, Brepols («Sous la Règle de saint Augustin»), 1998, p. 226. On trouvera une citation plus longue avec en note le texte latin (éd. Ilario Tolomio, Padoue, Antenore, 1975, p. 60) dans Ralph Dekoninck, «Alice au pays des mystiques. L'expérience du seuil dans la peinture religieuse entre Moyen Âge et Temps modernes", Cadre, seuil, limite. La question de la frontière dans la théorie de l'art, éd. Thierry Lenain et Rudy Steinmetz, Bruxelles, La Lettre volée («Essais»), 2010, p. 101-120, ici p. 114.

56. R. Dekoninck, ibid. 
et causé par Dieu, soit pour augmenter la dévotion, soit pour que l'âme quelque peu faible s'attache à un appui et ne s'égare pas, souvent c'est l'œuvre du démon qui veut tromper et nuire ${ }^{57}$.

La méfiance n'était cependant pas générale, tant s'en faut. Comme Jacqueline Jung l'a encore tout récemment montré à propos des sculptures religieuses des $\mathrm{XIII}^{\mathrm{e}}, \mathrm{XIV}^{\mathrm{e}}$ et $\mathrm{XV}^{\mathrm{e}}$ siècles, on considérerait souvent de façon positive l'expérience visionnaire d'une mobilité de l'image favorisée par l'interaction entre, d'une part, sa visibilité, voire sa tangibilité, et, d'autre part, une fervente imagination $^{58}$. Aux exemples relevés par Jung on ajoutera celui d'une des Cantigas de Santa Maria, composées à l'initiative et sous la direction d'Alphonse X, roi de Castille-León (1252-1284). Le roi avait offert une statue miraculeuse de Marie au monastère royal de La Huelgas à Burgos habité par des cisterciennes. Après avoir dit que Dieu avait mis en elle un grand pouvoir, la cantiga affirme «que la Reine spirituelle agissait par son image comme si elle était de chair». Une nuit de Noël, les moniales avaient couché la statue dans un lit richement paré comme pour une femme qui va accoucher. Tandis qu'elles la regardaient avec ravissement, elles virent son visage changer de couleur et son corps se tourner d'un côté à l'autre. La manipulation de l'effigie sacrée, la croyance en son pouvoir miraculeux et le contexte même nourrissaient l'imagination des nonnes ${ }^{59}$.

Cela nous conduit à un aspect bien connu que je ne puis rappeler qu'en quelques mots. Si l'imagination pouvait donner vie ou apparence de vie aux images, elle était souvent stimulée par leurs manipulations rituelles. Immobile, lointaine ou cachée, placée dans un dispositif marquant sa transcendance, l'image de culte était activée lors de dévoilements et de processions non seulement par

57. Jean de la Croix, La Montée du Mont Carmel, III, 36, présentation et traduction par Françoise Aptel, Mariannick Caniou et Marie-Agnès Haussièttre, Paris, Éd. du Cerf («Sagesses chrétiennes»), 2010, p. 427, que je modifie légèrement. Texte original: San Juan de la Cruz, Subida del Monte Carmelo, éd. Jose Vicente Rodriguez, Madrid, Editorial de Espiritualidad («Logos,» 54), 2006 (4éd.), p. 428.

58. J. E. Jung, «The Tactile and the Visionary» (voir n. 41).

59. Cantiga 361, éd. Walter Mettmann, Alfonso X, el Sabio. Cantigas de Santa María, vol. 3, Madrid, Castalia, («Clásicos Castalia»), 1989, p. 232-233; trad. Kathleen Kulp-Hill, Songs of Holy Mary of Alfonso X, The Wise. A Translation of the Cantigas de Santa Maria, Tempe, Arizona, ACMRS («Medieval and Renaissance Texts and Studies», 173), 2000, p. 440-441. 
son apparition et sa mobilité, mais aussi par l'attente et la ferveur des fidèles. Beaucoup de statues étaient vêtues; certaines étaient articulées, ce qui pouvait faire crier à l'imposture, mais était connu et admis par un nombre croissant de gens sans empêcher pour autant la ferveur. D'ingénieux mécanismes élevaient des effigies mariales lors de l'Assomption; des images étaient présentes aux côtés d'acteurs humains lors de représentations liturgiques ou paraliturgiques ${ }^{60}$. Bref, les manipulations et déjà l'échange de regards entre l'effigie et le dévot animaient l'image, contribuaient en quelque sorte à la transformer en une personne. C'est ce qu'observe de façon critique Lodovico Antonio Muratori vers 1747:

En contemplant une statue d'un saint pourvue avec art de tous les traits d'une personne vivante, son [= du peuple grossier] imagination peut la prendre pour une chose animée. Cette illusion s'ancre d'autant plus en lui qu'il voit cette statue portée en procession sous un baldaquin, courtisée par le clergé avec une grande quantité de cierges et d'encensoirs.

Muratori note ensuite que l'illusion de présence est d'autant plus grande qu'on rend au simulacre des honneurs quasi semblables à ceux rendus au Saint-Sacrement, à la présence réelle de $\mathrm{Dieu}^{61}$.

Il reste que beaucoup de miracles d'animation, surtout ceux à l'origine d'un culte, étaient étrangers à ces manipulations rituelles qui concernaient des images déjà vénérées. Un bel exemple est celui de Santa Maria delle Carceri à Prato, une fresque du milieu du $\mathrm{XIV}^{\mathrm{e}}$ siècle représentant la Vierge à l'Enfant flanquée par les saints

60. Outre les ouvrages mentionnés à la n. 48, cf. entre autres Susan Verdi Webster, Art and Ritual in Golden-Age Spain, Princeton (N.J.), Princeton University Press, 1993; Ead., "Shameless Beauty and Worldly Splendor. On the Spanish Practice of Adorning the Virgin», The Miraculous Image, p. 249-271; Valeria E. Genovese, Statue vestite e snodate. Un percorso, Pisa, Edizioni della Normale («Scuola Normale Superiore. Tesi », 6), 2011, et, à propos notamment de cet ouvrage, Marlène Albert Llorca, «Les statues habillées dans le catholicisme. Entre histoire de l'art, histoire religieuse et anthropologie», Archives de sciences sociales des religions, 164, 2013, p. 11-23. Je remercie l'auteure de m'avoir communiqué cette note critique et m'avoir ainsi fait connaître le riche livre de $\mathrm{V}$. E. Genovese.

61. Lodovico Antonio Muratori, Della regolata devozione dei cristiani, $1^{\text {re }}$ éd. Venise, Giambattista Albrizzi, 1747, republiée, avec en notes les variantes du manuscrit autographe et de sa copie ainsi que les remaniements faits ensuite par l'auteur, et introduite par Pietro Stella, Cinisello Balsamo, Edizioni Paoline («Storia della Chiesa / Fonti»), 1990. Chap. XxıII, Della divozione alle reliquie ed immagini dei santi, variante du manuscrit original autographe, éd. Stella, p. 256-257. 
Léonard et Étienne. L'image est encore vénérée dans l'église qui fut édifiée peu après les événements; auparavant, elle se trouvait audessus d'une fenêtre d'une prison abandonnée. En 1484, un garçon de huit ans vit la figure de la Vierge descendre du mur en s'en détachant, déposer son fils sur le sol et l'adorer. Les jours, les mois et les années suivantes, la Vierge - et la Vierge c'est Nostra Donna delle Charcere della terra di Prato - fit devant divers témoins des mutationi, des transfigurationi, soigneusement enregistrées, consistant surtout en des mouvements des yeux et des changements de couleur du visage; autrement dit, et cela n'a rien d'étonnant, l'expérience collective fut plus simple que celle de l'enfant. À cela s'ajoutèrent de nombreuses guérisons miraculeuses en liaison avec l'image ou ses figures de papier et de plomb ${ }^{62}$.

Robert Maniura a étudié les relations de ce culte naissant avec celui de la ceinture de la Vierge vénérée dans la cathédrale de Prato depuis le $\mathrm{XII}^{\mathrm{e}}$ ou le $\mathrm{XIII}^{\mathrm{e}}$ siècle. Il conclut que ni la ceinture ni la peinture n'étaient identifiées à la Vierge elle-même; elles laissaient entrevoir l'autre monde, mais elles auraient été en définitive les marques d'une absence ${ }^{63}$. Qu'il n'y ait pas eu confusion avec le prototype semble évident, mais le propos me paraît devoir être nuancé. Une relique aussi importante que la ceinture assurait la présence réelle de la Vierge qui le montra un jour en animant la statue de marbre posée sur l'autel de la Sainte-Ceinture ${ }^{64}$. Quant à la Madone delle Carceri, elle était à la fois «la»Vierge et «une» Vierge selon une ambiguïté intrinsèque dont j'ai parlé ailleurs à la

62. Deux manuscrits de Prato offrent une documentation exceptionnelle sur les miracles et le début du culte de l'image, l'un, copié à la fin du XV siècle avec des additions de 1514, contient un texte anonyme composé pour l'essentiel entre 1484 (date des premiers miracles) et 1487; l'autre est constitué des Miracoli rédigés par le juriste Giuliano di Francesco Guizzelmi qui termina son œuvre en 1505. La publication intégrale de ces textes est récente: Isabella Gagliardi, «I miracoli della Madonna delle Carceri in due codici della Biblioteca Roncioniana di Prato », Santa Maria delle Carceri a Prato. Miracoli e devozione in un santuario toscano del Rinascimento, éd. Anna Benvenuti, Florence, Mandragore, 2005, p. 97-153. Outre ce livre, on verra Robert Maniura, «The Images and Miracles of Santa Maria delle Carceri», The Miraculous Image, p. 81-95 (cf. aussi l'article mentionné à la note suivante); M. Holmes, The Miraculous Image in Renaissance Florence, passim.

63. R. Maniura, «Image and Relic in the Cult of Our Lady of Prato», Images, Relics, and Devotional Practices in Medieval and Renaissance Italy, éd. Sally J. Cornelison et Scott B. Montgomery, Tempe, Arizona, ACMRS («Medieval and Renaissance Texts and Studies», 296), 2006, p. 193-212.

64. Éd. I. Gagliardi, «I miracoli della Madonna delle Carceri», p. 135 (col. 2). 
suite de Marlène Albert Llorca ${ }^{65}$. La chronique de ses miracles relate qu' après la pose de la première pierre du nouveau sanctuaire marial, les cloches de l'église paroissiale et celle de Santa Maria in Castello sonnèrent à la louange «de Dieu tout puissant et della sua Gloriosa Madre sempre Vergine Maria delle Charcere $»^{66}$. Sa mère, Maria delle Carceri! Selon un poème contemporain, la Vierge descendant du ciel vint dans l'image, s'en détacha, puis retourna dans le mur où elle fit de grands miracles ${ }^{67}$. La Vierge est à la fois la même et autre que son image.

Souvent la documentation sur les origines du culte des images miraculeuses n'est pas aussi proche de l'événement; elle comprend nombre de traditions dont l'utilisation s'avère délicate, hormis pour l'époque où elles ont été mises par écrit et en ce qui concerne la typologie des légendes et des miracles pour lesquels les grandes compilations mariales du $\mathrm{XVII}^{\mathrm{e}}$ siècle sont particulièrement précieuses. C'est, du reste, une typologie que fait à sa manière le jésuite Wilhelm Gumppenberg dans la deuxième édition de l'Atlas Marianus publiée en 1672, une œuvre qui réussit le tour de force de valoriser les cultes locaux des effigies mariales miraculeuses tout en les ramenant au prototype unique alors que les protestants soulignaient l'incompatibilité entre la pluralité des images de culte et l'unicité du modèle céleste. L'ouvrage comprend une peritia, une «connaissance acquise par l'expérience», des images miraculeuses. Il s'agit d'une sorte d'immense index raisonné et commenté. Les multiples aspects du prototype unique, la figure mariale universelle, se déclinent de façon variée dans ses diverses images locales et sont systématiquement répertoriés dans douze chapitres eux-mêmes divisés en plusieurs catégories. Un chapitre concerne les modes d'action de la Vierge, agissant tantôt par elle-même, tantôt par le

65. J.-M. Sansterre, «Unicité du prototype et individualité de l'image : la Vierge et ses effigies miraculeuses, approche diachronique d'une croyance entre évidence, rejet et ambiguïté», Image et prototype, éd. Thierry Lenain, J.-M. Sansterre et Ralph Dekoninck, Bruxelles, $2011=$ Degrés. Revue de synthèse à orientation sémiologique, 145-146, p. F1-17. Cf. M. Albert Llorca, Les Vierges miraculeuses, en particulier p. 70.

66. Éd. I. Gagliardi, «I miracoli della Madonna delle Carceri», p. 126 (col. 1).

67. Passage du poème de Lorenzo di Jacopo degli Obbizzi (incunable, c. 1485) cité et commenté - en allant peut-être au-delà de ce que dit le texte - par Megan Holmes, «Miraculous Images in Renaissance Florence», Art History, 34, 2011, p. 432-465, ici p. 452-453, 464; cf. aussi Ead., The Miraculous Image in Renaissance Florence, p. 182-183, 326. 
biais de statues, le catalogue distinguant les paroles des œuvres. Voici ce que Gumppenberg dit sous la rubrique verba:

Ici j'indique certaines statues miraculeuses de la Mère de Dieu qui (sont des statues) parlantes. De la sorte, si les hommes taisent la gloire de la Vierge Mère, les pierres et les bois parlent. Je vais répartir ce catalogue en séries de celles qui avec une voix humaine et distinctement ont salué, se sont plaintes, ont pleuré, ont crié, ont pleuré des larmes de sang, ont chanté, ont approuvé, ont souri, ont réclamé un sanctuaire, et ont prononcé des mots de quelque autre manière,

l'auteur renvoyant chaque fois aux numéros des images concernées ${ }^{68}$. De même pour les opera:

Je ne sais si les actes sont plus merveilleux que les paroles. Albert le Grand, à en croire la renommée, fit des statues parlantes, non pas marchant ou accomplissant un autre acte d'un homme vivant ${ }^{69}$. Je vais répartir ce catalogue en séries de celles qui bougent les yeux, ouvrent les yeux, présentent la main, menacent du doigt, cèdent le pas à une autre statue, se rendent invisibles aux ennemis, descendent d'un mur, déchirent leurs parures, déchirent (leurs) vêtements, lèvent le pied, laissent l'empreinte de leurs pas, marchent, tournent le dos, changent leur sein, changent leur visage, arrêtent leurs porteurs, s'avancent contre les ennemis, inclinent la tête, courbent le doigt, écartent les doigts, descendent d'un autel, reçoivent des anneaux, reçoivent des supplications écrites, refusent de revenir ${ }^{70}$.

Ce que Gumppenberg affirme des images parlantes et agissantes doit être pris au sérieux: pour lui, la parole et le mouvement étaient deux des multiples traits que la Vierge, regardée sans réserve comme l'auteure de ses miracles, distribuait entre ses images ${ }^{71}$.

J'ignore s'il est possible d'étudier la réception de tels propos parmi les catholiques du XVII ${ }^{\mathrm{e}}$ siècle, mais il ne s'agit certainement

68. Guilielmus (Wilhelm) Gumppenberg, Atlas Marianus quo Sanctae Dei Genitricis Mariae Imaginum Miraculosarum Origines Duodecim Historiarum Centuriis explicantur, Munich, Johannes Jaecklin, 1672 : Peritia Atlantis Mariani, caput XI, index II, catalogus I (sans pagination). Sur cet ouvrage, on verra désormais Marie mondialisée. L'Atlas Marianus de Wilhelm Gumppenberg et les topographies sacrées de l'époque moderne, dir. Olivier Christin, Fabrice Flückiger, Naïma Ghermani, Neuchâtel, Éditions Alphil, Presses universitaires suisses, 2014. Sur la Peritia, voir dans le présent volume l'article de Ralph Dekoninck.

69. Selon un récit légendaire fort répandu, Albert le Grand aurait forgé en trente ans de travail un automate parlant que Thomas d'Aquin aurait détruit: Alain de Libera, Métaphysique et noétique: Albert le Grand, Paris, Vrin, («Problèmes et controverses»), 2005, p. 20.

70. Peritia Atlantis Mariani, caput XI, index II, catalogus II.

71. Dans le même sens, à propos du frontispice de l'Atlas Marianus: J. Garnett et G. Rosser, Spectacular Miracles, p. 11-13 avec la photo p. 10. 
pas d'un combat d'arrière-garde. Ainsi, il était tout à fait concevable pour une partie des catholiques qu'une image sainte pût parler. Un article récent de Luis Corteguera a attiré l'attention sur un recueil inédit de soixante-dix-neuf histoires relatives à des images parlantes, surtout du Christ et de la Vierge, rassemblée avec dévotion par un érudit catalan, dans les années $1720^{72}$.

Bien sûr, il avait aussi des sceptiques ou tout simplement des modérés enclins à une prudente réserve, tel l'abbé Michel de Marolles. Dans ses mémoires publiées en 1656, de Marolles raconte un fait survenu à Paris en 1644. On avait rapporté à la Princesse Marie de Nevers, dont «le naturel doux a toujours été facile à croire les miracles», «qu'une enseigne du Pont Notre-Dame, où il y avait une Vierge peinte, avait versé du sang, d'une blessure qu'un impie, ou un hérétique, lui avait faite en déchargeant un pistolet». La princesse en était persuadée.

Je lui assurai [dit de Marolles] que cela n'était point, et qu'il ne pouvait être dans les desseins de Dieu, qui ne fait de miracles que pour autoriser quelque vérité importante qui tende à sa gloire, ce qui ne se voyait pas ici, où le miracle ne servirait de rien ». [Mais, poursuit notre abbé] «cela ne fut pas capable de l'empêcher de croire la déposition de force gens, qui lui en parlaient tous comme témoins oculaires, ajoutant que cinquante mille personnes l'avaient vu comme eux. De sorte que pour la contenter, après avoir dit qu'il fallait tenir pour maxime, qu'en matière de superstition, le peuple ne voit même pas ce qu'il regarde, je m'en allai sur les lieux pour m'en informer plus exactement. Et il se trouva bien qu'on avait tiré un coup de pistolet dans l'enseigne, sans y penser, mais tout le reste était fabuleux, en quoi je ne fus nullement trompé $e^{73}$.

Outre l'attitude de l'abbé, on retiendra la facilité de la propagation de ce qui pour beaucoup de gens - y compris la princesse - était bel et bien un miracle, d'autant plus aisé à accepter qu'il s'inscrivait dans une longue tradition de prodiges de ce genre.

Tout cela ne constitue bien sûr qu'un aperçu d'un sujet aussi difficile que passionnant. Peut-être faudrait-il le nuancer sur un

72. Luis R. Corteguera, «Talking Images in the Spanish Empire: Vision and Action», Visual Resources, 25, 2009, p. 53-68.

73. Mémoires de Michel de Marolles, abbé de Villeloin, divisez en trois parties, contenant ce qu'il a vu de plus remarquable en sa vie depuis l'année 1600, 2 vol., Paris, Antoine de Sommaville, 1656 [en ligne: Gallica], vol. I, p. 153. Je modernise l'orthographe et la ponctuation. 
point important pour ne pas faire apparaître le miracle d'image plus exceptionnel qu'il ne pouvait l'être en «réalité». Il devait, en effet, exister des attitudes plus ou moins proches de ce que l' anthropologue Francesco Faeta a observé en milieu populaire calabrais à propos de l'animation des effigies sacrées. Qu'ils aient lieu la nuit en rêve ou durant le jour, les mouvements des yeux, du visage, des bras, la sueur, les larmes, les changements d'expression sont des faits courants, normaux, et non des événements extraordinaires comme pour le clergé ${ }^{74}$. Sur un plan plus large, on rappellera, avec Jane Garnett et Gervase Rosser, que le monde des images miraculeuses, lors du plein épanouissement de leur culte, est «un monde dans lequel la présence du surnaturel, médiatisé par des copies d'images particulièrement saintes, fait intimement partie de la vie quotidienne de la famille et de la communauté» ${ }^{75}$.

Université Libre de Bruxelles [ULB]

UR SOCIAMM

Histoire CP 175

Avenue F.D. Roosevelt, 50

B-1050 Bruxelles

74. Francesco Faeta, "Qualche appunto sulle immagini e la santità in un contesto popolare moderno», Santità, culti, agiografia. Temi e prospettive, éd. Sofia Boesh Gajano, Rome, Viella, 1997, p. 241-253, ici p. 245-246.

75. J. Garnett et G. Rosser, Spectacular Miracles, p. 169. 